\title{
Correlazioni ed analisi fattoriale fra le varie manifestazioni cliniche della diatesi mesenchimosica in rapporto al fattore età
}

\author{
V. Bartoli, G. G. Neri Serneri, I. Nicoletti
}

L'osservazione clinica ha da tempo permesso di rilevare un'alta incidenza di associazione in atto od anamnestica fra alcuni quadri anatomo-clinici quali l'artrosi ad impronta diffusiva, le varici, le ernie, alcune manifestazioni di fragilità venulare (epistassi giovanile recidivante ed i suoi equivalenti ed ecchimosi spontanee), le ptosi viscerali, gli stati di lassità legamentosa ecc.

Questo vasto complesso è stato da Lunedei riunito sotto il termine comprensivo di mesenchimosi. Fsso è solo in parte sovrapponibile allo «Status varicosus » di Curtius.

In precedenti ricerche 1 abbiamo potuto dimostrare che l'associazione fra artrosi, varici, ernia e manifestazioni di fragilità venulare è statisticamente valida poichè supera di gran lunga la incidenza di ciascuna di queste forme nella popolazione generale. Questo esclude che l'associazione sia dovuta ad una pura casualità. Ciò trova conferma anche nelle ricerche di ordine familiare che hanno messo in rilievo sia una familiarità per ciascuna delle forme considerate, sia soprattutto una familiarità per tutto il complesso preso in esame.

Tuttavia la validità statistica dell'associazione fra $i$ vari quadri anatomo-clinici da noi presi in esame potrebbe essere infirmata se si dimostrasse che l'associazione stessa è dovuta all'influsso dell'età. L'osservazione clinica dimostra infatti che i quadri clinici considerati tendono generalmente a manifestarsi verso il quarto-quinto decennio e soprattutto tendono ad aggravarsi con il progredire dell'età, per cui si potrebbe ritenere che l'età fosse il fattore capace di condizionare da solo la comparsa e l'associazione di queste manifestazioni. Un tale modo di vedere può trovare sostegno nella conoscenza che i fenomeni regressivi dei tessuti connettivi - ai quali sembra verosimile riportare, quale ultimo anello della catena patogenetica, queste diverse manifestazioni - si instaurano come naturale conseguenza del fisiologico processo di invecchiamento, con conseguente maggiore facilità d'insorgenza di alcuni quadri morbosi in determinate epoche della vita.

1 Neri Serneri G. G. e Bartoli V.: Fondamenti genetici della diatesi mesenchimosica (A.Ge.Me.Ge. 7. 101 e 266,1958 ). 
Un'altra considerazione che ci spinge a ricercare l'influenza dell'età nel determinare questa associazione sintomatologica deriva dal fatto che da alcuni AA. sono state avanzate critiche allo status varicosus di Curtius del quale fanno parte alcune delle manifestazioni della diatesi mesenchimosica. Sono ben note le polemiche sul valore dei dati raccolti da Curtius (polemica che particolarmente aspra si svolse con Siemens e con il suo allievo Beek) per cui anche alcuni valorosi genetisti, quali Weitz e Lenz, giunsero a negare la reale esistenza dello status varicosus. Del tutto recentemente Wagner ha affrontato di nuovo il problema dell'esistenza dello status varicosus ricercando la validità statistica dell'associazione fra alcune delle più frequenti manifestazioni di questo (teleangiectasie essenziali, varici ed angiomi senili), dopo eliminazione dell'influsso dell'età. In base ai suoi risultati Wagner ritiene che « lo status varicosus sia solo una costruzione teorica alla quale non corrisponde una realtà clinica ", poichè l'associazione delle singole manifestazioni è condizionata dal fattore età, dato che con il progredire di questa si ha una maggior frequenza delle singole manifestazioni e quindi un'apparente associazione.

In una precedente indagine ${ }^{2}$ cercammo di valutare l'importanza del fattore età nel determinare il grado di associazione di alcune manifestazioni della diatesi mesenchimosica, ripromettendoci di riprendere l'argomento allorchè fossimo stati in possesso di dati più completi e tali da permetterci elaborazioni più approfondite.

In questa nota, mediante l'impiego del coefficiente di correlazione semplice, del coefficiente di correlazione parziale e dell'analisi fattoriale, viene ricercata l'influenza che il fattore età può avere nella comparsa e, soprattutto, nell'associazione di alcuni quadri anatomo-clinici, quali l'artrosi ad impronta diffusiva, le varici, le ernie, l'epistassi giovanile recidivante e le ecchimosi spontanee, allo scopo di stabilire se questa loro associazione sia condizionata da un fondamento patogenetico comune oppure se essa non sia che un'evenienza legata al progredire dell'età.

\section{Raccolta dei dati}

Questa indagine è stata condotta su $4^{\mathrm{I}} 3$ soggetti, ${ }_{5} 6$ uomini e 257 donne, ricoverati consecutivamente nell'Istituto di Patologia Medica e di Semeiotica Medica. Ogni soggetto veniva interrogato ed esaminato per accertare la presenza di artrosi, di varici, di ernia e di manifestazioni di fragilità venulare (epistassi ed ecchimosi spontanee). Per quei casi nei quali l'esame clinico non permetteva di porre con sicurezza la diagnosi di artrosi si procedeva all'indagine radiologica.

I criteri diagnostici da noi seguiti nella presente indagine sono gli stessi che ci hanno guidato nelle precedenti ricerche, alle quali pertanto rimandiamo.

Per ognuna delle manifestazioni in esame veniva annotata l'età di insorgenza quale risultava dall'interrogatorio del paziente.

Nella tabella I è riportata la distribuzione per età dei soggetti al momento dell'in-

2 Neri Serneri G. G., Bartoli V. e Nicoletti I. : Analisi statistica e considerazioni sull'importanza dell'età per l'associazione di varie manifestazioni dello stato mesenchimosico. Riv. Clin. Med. $195^{8}$ (in corso di stampa). 
Bartoli V., Neri Serneri G. G., Nicoletti I.: Correlazioni ed analisi fattoriale, ecc.

dagine; nella tabella 2 viene invece illustrata la frequenza delle varie manifestazioni secondo l'età d'insorgenza. In quest'ultima tabella non è stata riferita l'età d'insorgenza delle manifestazioni di fragilità venulare perchè la loro comparsa è generalmente limitata a determinate epoche della vita e particolarmente all'età prepuberale e puberale per l'epistassi e alla fase sessualmente attiva della donna per le ecchimosi spontanee.

Tabella I - Frequenze dei soggetti esaminati e delle manifestazioni mesenchimosiche secondo l'età

\begin{tabular}{|c|c|c|c|c|c|c|c|c|c|c|}
\hline & & \multicolumn{9}{|c|}{ ETA } \\
\hline & & IO- & $20-$ & $30-$ & $40-$ & $5^{\circ-}$ & $60-$ & $70-$ & 80- & Totale \\
\hline \multirow{3}{*}{ Soggetti esaminati } & $\sigma^{x}$ & 6 & 7 & Io & 43 & $5^{2}$ & $3 \mathbf{I}$ & 7 & - & I 56 \\
\hline & & 24 & 39 & $5^{8}$ & 57 & 44 & 29 & 5 & I & 257 \\
\hline & Totale & $3^{\circ}$ & $4^{6}$ & 68 & IOO & 96 & 60 & 12 & I & 413 \\
\hline \multirow{3}{*}{ Artrosi } & $\sigma^{x}$ & - & - & I & I 2 & 13 & I3 & 4 & - & 43 \\
\hline & ? & - & $\mathbf{I}$ & I 5 & I 7 & 22 & IO & 2 & - & 67 \\
\hline & Totale & - & I & 16 & 29 & 35 & 23 & 6 & 一 & I IO \\
\hline \multirow{3}{*}{ Varici } & $\sigma$ & - & I & 3 & I I & I3 & 9 & 4 & $\longrightarrow$ & $4 \mathbf{I}$ \\
\hline & 9 & 2 & 6 & I 6 & I3 & 13 & IO & I & 一 & $6 I$ \\
\hline & Totale & 2 & 7 & 19 & 24 & 26 & I9 & 5 & - & 102 \\
\hline \multirow{3}{*}{ Ernia } & $0^{x}$ & - & 2 & I & 10 & 12 & 7 & 2 & $\cdots$ & 34 \\
\hline & 千 & I & I & 5 & 6 & 2 & I & - & - & 16 \\
\hline & Totale & I & 3 & 6 & I 6 & 14 & 8 & 2 & - & $5^{\circ}$ \\
\hline \multirow{3}{*}{$M$ f v } & $\sigma^{\top}$ & I & 5 & 2 & 17 & I 4 & 9 & 3 & - & $5^{I}$ \\
\hline & 9 & 8 & I 4 & 28 & 25 & I9 & 7 & 3 & - & IO4 \\
\hline & Totale & 9 & 19 & 30 & $4^{2}$ & 33 & I6 & 6 & - & ${ }^{155}$ \\
\hline
\end{tabular}

Tabella 2 - Frequenze delle manifestazioni mesenchimosiche secondo l'età in cui si sono rese clinicamente manifeste

\begin{tabular}{|c|c|c|c|c|c|c|}
\hline \multirow{2}{*}{ Età } & \multicolumn{3}{|c|}{ Uomini } & \multicolumn{3}{|c|}{ Donne } \\
\hline & Artrosi & Varici & Ernia & Artrosi & Varici & Ernia \\
\hline $0-9$ & - & - & 2 & - & - & - \\
\hline $10-19$ & - & - & 2 & I & 2 & I \\
\hline $20-29$ & 3 & 2 & 4 & 5 & I3 & 2 \\
\hline $3^{0}-39$ & 7 & 9 & 8 & 21 & 25 & 9 \\
\hline $40-49$ & 13 & 15 & 9 & 24 & 12 & 4 \\
\hline $50-59$ & 17 & I 1 & 8 & I2 & 6 & - \\
\hline $60-69$ & 3 & 4 & I & 4 & 3 & - \\
\hline Totale & 43 & $4^{1}$ & 34 & 67 & $6 I$ & I6 \\
\hline
\end{tabular}




\section{Elaborazione dei dati}

Prima di esaminare l'influenza dell'età sull'associazione delle manifestazioni considerate, abbiamo voluto esaminare, per mezzo del calcolo del coefficiente di correlazione semplice, il grado di relazione esistente fra le manifestazioni stesse. Ciò anche allo scopo di avere una conferma ai risultati ottenuti nella precedente ricerca su materiale raccolto con criteri diversi. Il coefficiente di correlazione (indice di Bravais-Pearson o indice $r$ ) è stato calcolato sulla base della formula seguente:

$$
\mathrm{r}=\frac{\sum \mathrm{xy}-\frac{\Sigma \mathrm{x} \cdot \Sigma \mathrm{y}}{\mathrm{n}}}{\sqrt{\left(\sum \mathrm{x}^{2}-\frac{(\Sigma \mathrm{x})^{2}}{\mathrm{n}}\right)\left(\Sigma \mathrm{y}^{2}-\frac{(\Sigma \mathrm{y})^{2}}{\mathrm{n}}\right)}}
$$

ove $r$ indica il coefficiente di correlazione; $x$ ed $y$ i due caratteri posti in relazione fra loro; $\Sigma x y$ la somma di tutti i prodotti $x y ; \Sigma x$ e $\Sigma y$ la somma rispettivamente degli $x$ e degli $y ; n$ il numero dei soggetti esaminati; $\Sigma x^{2}$ e $\Sigma y^{2}$ la somma rispettivamente degli $x$ e degli $y$ elevati al quadrato. Ricordiamo che in base alle tavole di Fisher e Yates ${ }^{3}$ per $n=$ ıoo il valore di $r=0$, I946 è significativo con $\mathrm{P}=0,05$; il valore di $r=0,254^{\circ}$ è significativo con $\mathrm{P}=0, \mathrm{OI}$; il valore di $r=0,32 \mathrm{I}$ I è significativo con $\mathrm{P}=0,00 \mathrm{I}$.

Nel nostro materiale il calcolo del coefficiente di correlazione è stato eseguito separatamente nei due gruppi di soggetti, uomini e donne, per ciascuna combinazione a due a due dei quadri anatomo-clinici in esame (artrosi-varici; artrosi-ernia; artrosimanifestazioni di fragilità venulare ecc.).

I risultati vengono riferiti nella tabella 3 .

Tabella 3 - Coefficienti di correlazione semplice fra le manifestazioni mesenchimosiche

\begin{tabular}{|c|c|c|c|c|c|}
\hline & & artrosi & varici & ernia & man. frag. venul. \\
\hline artrosi & $\begin{array}{l}x \\
7\end{array}$ & & $\begin{array}{l}0,4790 \\
0,4603\end{array}$ & $\begin{array}{l}0,265 \mathrm{I} \\
0,2872\end{array}$ & $\begin{array}{l}0,4264 \\
0,4855\end{array}$ \\
\hline varici & $\begin{array}{l}7 \\
7\end{array}$ & $\begin{array}{l}0,4790 \\
0,4603\end{array}$ & & $\begin{array}{l}0,3197 \\
0,1969\end{array}$ & $\begin{array}{l}0,2929 \\
0,4344\end{array}$ \\
\hline ernia & $\begin{array}{l}x \\
9\end{array}$ & $\begin{array}{l}0,2651 \\
0,2872\end{array}$ & $\begin{array}{l}0,3^{197} \\
0,19^{69}\end{array}$ & & $\begin{array}{l}0,3603 \\
0,3125\end{array}$ \\
\hline manif. frag. ven. & 8 & $\begin{array}{l}0,4264 \\
0,4855\end{array}$ & $\begin{array}{l}0,2929 \\
0,4344\end{array}$ & $\begin{array}{l}0,3603 \\
0,3 \mathbf{I} 25\end{array}$ & \\
\hline
\end{tabular}

Totale dei soggetti: 156 uomini e 257 donne.

3 Fisher A. R. e Yates F.: Statistical tables. Oliver \& Boyd. London, 1953. 
Bartoli V., Neri Serneri G. G., Nicoletti I.: Correlazioni ed analisi fattoriale, ecc.

Negli uomini ogni manifestazione appare correlata significativamente $(\mathrm{P} \ll \mathrm{O}, \mathrm{OI})$ con ciascuna delle altre prese in esame.

Infatti, la forza della correlazione non è identica in tutte le combinazioni: così ad es. per l'artrosi si osserva che il legame maggiore esiste con le varici e con le manifestazioni di fragilità venulare (rispettivamente $r=0,47$ e 0,42 ); per le varici con l'artrosi $(r=0,47)$; per l'ernia con le manifestazioni di fragilità venulare $(r=0,36)$ e per le manifestazioni di fragilità venulare con l'artrosi $(r=0,42)$.

Per quanto concerne il gruppo delle donne (tab. 3) può osservarsi che il calcolo fornisce risultati significativi $(\mathrm{P}\langle\mathrm{o}, \mathrm{oI})$ per tutte le correlazioni ad eccezione di quella varici-ernia che mostra un valore di $r$ al limite della significatività. In particolare, come è illustrato nella tabella 3 , l'artrosi e le manifestazioni di fragilità venulare, che sono correlate in maniera significativa con ciascuna delle altre manifestazioni, appaiono correlate più strettamente fra di loro. $(r=0,48)$; le varici e l'ernia, che sono correlate in maniera significativa con l'artrosi e le manifestazioni di fragilità venulare (rispettivamente $r=0,46 \mathrm{e} 0,43$ per le varici, e $r=0,28 \mathrm{e} \mathrm{o,3} \mathrm{I}$ per l'ernia) presentano invece fra loro una correlazione al limite della significatività $(r=0$, I 969$)$. Anche in questo gruppo pertanto i risultati forniti dal calcolo del coefficiente di correlazione dimostrano che fra ciascuno dei caratteri in esame esiste una reciproca relazione. Questi dati depongono pertanto per l'esistenza di una correlazione positiva fra $i$ vari quadri clinici studiati.

In base ad elementi di vario ordine derivanti dall'osservazione clinica ed in parte deducibili dalla letteratura, le correlazioni trovate potrebbero essere interpretate come la risultante dell'influenza dell'età su ciascun carattere. Un esame sommario del materiale raccolto (tab. I) mette in evidenza che le varie forme anatomo-cliniche, ad esclusione delle manifestazioni di fragilità venulare, tendono a divenire più frequenti, almeno entro certi limiti, con il progredire dell'età. Questo fatto, che è particolarmente evidente per l'artrosi, è presente, sia pure in grado minore, per le varici e per l'ernia. Pertanto la maggiore incidenza dell'artrosi, ed in linea subordinata delle varici e dell'ernia, nelle classi di età più avanzate pone in discussione se le correlazioni precedentemente dimostrate non debbano essere interamente attribuite all'età.

Un primo elemento per la risoluzione del problema dei rapporti fra età e manifestazioni dello stato mesenchimosico viene offerto dal calcolo del coefficiente di correlazione fra l'età da un lato e ciascuna manifestazione dall'altro.

I risultati forniti da questa indagine appaiono sufficientemente dimostrativi (tab. 4): l'età appare correlata solamente con l'artrosi $(r=0,30$ nelle donne e 0,26 negli uomini) .

Tabella 4 - Coefficienti di correlazione fra età e manifestazioni mesenchimosiche

\begin{tabular}{l|c|c}
\hline \hline & & E T A \\
& uomini & donne \\
artrosi & 0,2630 & 0,3059 \\
varici & 0,2033 & 0,1332 \\
ernia & 0,0786 & 0,0682 \\
manif. frag. ven. & 0,0453 & 0,0296
\end{tabular}


Per quanto concerne le altre manifestazioni, soltanto nel gruppo degli uomini è possibile rilevare per le varici un coefficiente di correlazione al limite della significatività statistica $(r=0,20)$.

Dall'esame dei risultati di questa ricerca sembra giustificato ritenere che l'età non influenzi in maniera significativa l'insorgenza di tutte le manifestazioni in esame e quindi anche la loro associazione, poichè essa appare significativamente correlata soltanto con uno dei quattro elementi considerati.

Una ulteriore più esatta valutazione dell'influenza dell'età sull'associazione dei quadri morbosi in esame è fornita dal calcolo del coefficiente di correlazione parziale che permette di saggiare la correlazione fra due "caratteri " dopo aver correttamente eliminato l'influenza di una terza dimensione che, nel nostro caso, è rappresentata dall'età.

Il calcolo viene eseguito mediante l'impiego della formula seguente:

$$
r_{1-2,3}=\frac{r_{1-2}-r_{1-3} \cdot r_{2-3}}{\sqrt{\left(1-r_{1-3}^{2}\right)\left(1-r_{2-3}^{2}\right)}}
$$

ove $I, 2,3$, indicano tre variabili distinte; i simboli $r_{1-2} r_{1-3} r_{2-3}$ corrispondono ai coefficienti di correlazione (indice di Bravais-Pearson) calcolati rispettivamente fra le variabili I-2, I-3, 2-3; il simbolo $r_{\mathrm{I}-2,3}$, indica il coefficiente di correlazione parziale fra le variabili i e 2 dopo aver eliminato l'influenza della variabile 3 . Nel nostro caso la variabile 3 corrisponde sempre all'età, la cui influenza desideriamo eliminare dalle intercorrelazioni fra $\mathrm{i}$ sintomi.

I risultati forniti da questo calcolo escludono che l'età possa essere l'elemento comune condizionante l'associazione dei quadri clinici studiati (tab. 5). Il valore del coefficiente di correlazione parziale, dopo eliminazione della variabile età, appare in tutti i casi molto vicino al corrispondente valore del coefficiente di correlazione semplice.

Tabella 5 - Coefficienti di correlazione parziale fra le manifestazioni mesenchimosiche

\begin{tabular}{|c|c|c|c|c|}
\hline & artrosi & varici & ernia & man. frag. ven. \\
\hline artrosi & & $\begin{array}{l}0,45 \\
0,45\end{array}$ & $\begin{array}{l}0,25 \\
0,32\end{array}$ & $\begin{array}{l}0,45 \\
0,52\end{array}$ \\
\hline varici & $\begin{array}{l}0,45 \\
0,45\end{array}$ & & $\begin{array}{l}0,3 \mathbf{I} \\
0,2 \text { I }\end{array}$ & $\begin{array}{l}0,3 \mathbf{I} \\
0,44\end{array}$ \\
\hline ernia & $\begin{array}{l}0,25 \\
0,32\end{array}$ & $\begin{array}{l}0,3 \mathrm{I} \\
0,2 \mathrm{I}\end{array}$ & & $\begin{array}{l}0,36 \\
0,31\end{array}$ \\
\hline manif. frag. ven. & $\begin{array}{l}0,45 \\
0,5^{2}\end{array}$ & $\begin{array}{l}o, 3 \mathbf{I} \\
0,44\end{array}$ & $\begin{array}{l}0,3^{6} \\
0,3^{1}\end{array}$ & \\
\hline
\end{tabular}


Bartoli V., Neri Serneri G. G., Nicoletti I.: Correlazioni ed analisi fattoriale, ecc.

Esiste quindi, anche dopo eliminazione dell'età, una correlazione significativa fra le varie manifestazioni.

$\mathrm{E}$ giustificato quindi ritenere che la correlazione fra le varie manifestazioni in esame sia sostenuta da un fattore patogenetico comune, sicuramente indipendente dall'età.

Una tale ipotesi può essere avvalorata dall'impiego dell'analisi fattoriale.

In un lavoro precedente ${ }^{4}$ riguardante l'analisi fattoriale nelle ricerche clinicoterapeutiche, è stato messo in evidenza che «l'analisi fattoriale è un metodo idoneo a ricercare l'esistenza di un fattore comune determinante l'associazione di alcuni caratteri nello stesso individuo ".

Nel presente lavoro il calcolo viene limitato alla ricerca del primo fattore comune.

Rimandando al lavoro citato per quanto riguarda $i$ fondamenti dell'analisi fattoriale e il metodo centroide di Thurstone, da noi impiegato, ricordiamo solo che con l'analisi fattoriale è possibile determinare anche la misura con cui l'eventuale fattore comune influisce sulla comparsa di ciascuno dei sintomi che si presentano associati fra loro. Si può così mettere in evidenza se esiste un fattore comune che condiziona l'associazione dei sintomi ed inoltre se i sintomi considerati sono tutti correlati in grado elevato con il fattore comune.

Il risultato dell'analisi fattoriale da noi eseguita è riportato nella tabella delle saturazioni in primo fattore comune (tab. 6).

Tabella 6 - Saturazioni in primo fattore comune

\begin{tabular}{l|c|c}
\hline \hline & uomini & donne \\
artrosi & 0,70 & 0,76 \\
varici & 0,65 & 0,63 \\
manif. frag. ven. & 0,54 & 0,63 \\
ernia & 0,51 & 0,39 \\
età & 0,28 & 0,24
\end{tabular}

Un esame delle saturazioni dimostra l'esistenza di un fattore che satura in grado elevato tutti $i$ sintomi presi in considerazione; questo fattore non è sicuramente l'età, poichè essa appare saturata in misura notevolmente inferiore a tutti gli altri elementi.

Un esame comparativo delle saturazioni riferentisi agli uomini e di quelle che riguardano le donne permette di osservare che il «carattere » ernia è saturato sempre in maniera significativa sia negli uomini che nelle donne, ma in grado più elevato nei primi. Se ne può dedurre che l'ernia, pur essendo strettamente correlata con il fattore comune condizionante l'associazione dei sintomi esaminati si ritrova più facilmente associata alle altre manifestazioni negli uomini che non nelle donne.

Anche il risultato di questa indagine matematica conferma pertanto l'esistenza di un fattore comune, al di fuori dell'età, al quale devono riportarsi le intercorrelazioni osservate fra i vari elementi della diatesi mesenchimosica.

4 Nicoletti I. e Magherini G.: L'analisi fattoriale nelle ricerche clinico-terapeutiche. Rass. st. psichiat. $46,903,1957$. 


\section{Discussione}

L'elaborazione statistica dei nostri dati indica che l'alta incidenza di associazione di alcuni quadri anatomo-clinici, quali l'artrosi ad impronta diffusiva, le varici, le ernie e le manifestazioni di fragilità venulare, non è condizionata dal progredire dell'età. Infatti la correlazione fra queste manifestazioni rimane statisticamente significativa anche dopo eliminazione dell'influsso dell'età ottenuta mediante il calcolo del coefficiente di correlazione parziale.

Una conferma a tale conclusione viene fornita dai risultati dell'analisi fattoriale che, mentre da un lato depongono per l'esistenza di un fattore comune responsabile dell'associazione dei vari quadri anatomo-clinici, dall'altro escludono che questo fattore possa essere rappresentato dall'età. Degno di interesse è lo studio dei rapporti fra età ed insorgenza dei vari segni della diatesi mesenchimosica. Dal calcolo del coefficiente di correlazione semplice appare chiaramente che solo l'artrosi può essere legata al progredire dell'età, ma non necessariamente a questo solo fattore, poichè essa appare ancor più strettamente collegata con le altre manifestazioni che non risentono dell'influenza dell'età. E chiaro quindi che l'associazione dell'artrosi con le altre manifestazioni del complesso mesenchimosico non è dovuta al fatto che essa diventa sempre più frequente con il progredire dell'età, ma a quel medesimo fattore patogenetico responsabile dell'insorgenza di tutte le altre manifestazioni.

Questa nostra indagine statistica conferma quindi quanto già l'osservazione clinica aveva consentito di rilevare e cioè che accanto ad un'artrosi più o meno legata alla fisiologica usura da invecchiamento dei tessuti cartilaginei, esiste un'artrosi nella quale i fenomeni regressivi e degenerativi della cartilagine insorgono quale espressione di uno stato di diffusa labilità connettivale. In questo caso con altissima incidenza si osserverà un'associazione in. atto o anamnestica dell'artrosi con le altre manifestazioni della diatesi mesenchimosica (Neri Serneri e Bartoli).

L'associazione dell'artrosi ad impronta diffusiva, delle varici, delle ernie e delle manifestazioni di fragilità venulare non è pertanto dovuta ad una evoluzione parallela di singole manifestazioni indipendenti, ma rappresenta l'espressione molteplice di un comune fattore patogenetico. Tale comune denominatore patogenetico, che sembra da identificarsi con una labilità connettivale ereditaria, può essere favorito nella sua estrinsecazione dal fisiologico invecchiamento dei tessuti, la cui importanza è però del tutto secondaria.

Un'altro dato che vogliamo sottolineare riguarda la diversa modalità di associazione delle varie manifestazioni mesenchimosiche nei due sessi. Risulta infatti dall'analisi del coefficiente di correlazione semplice che nelle donne l'ernia presenta con le altre manifestazioni una correlazione inferiore a quella osservata negli uomini e, per quanto riguarda la combinazione ernia-varici il coefficiente di correlazione risulta al limite di significatività. Questo fatto potrebbe indurre a ritenere che la manifestazione erniosa non partecipi alla diatesi mesenchimosica: l'analisi fattoriale ci dimostra invece che, sia pure con differenza nei due sessi, il sintomo ernia è sempre saturato in maniera elevata. Questo indica che l'ernia fa parte con sicurezza della diatesi mesenchimosica 
pur potendo presentarsi con frequenza inferiore rispetto alle altre manifestazioni, specialmente nel sesso femminile.

Sulla diversa modalità di associazione delle singole forme del complesso mesenchimosico abbiamo già richiamato l'attenzione in un nostro precedente studio sulla diatesi mesenchimosica, nel quale venivano discussi i diversi fattori che possono in vario modo condizionare la comparsa dell'una o dell'altra manifestazione mesenchimosica e le loro diverse modalità di combinazione. Queste ultime, oltre a differire nei due sessi, possono variare anche in rapporto all'età indicandoci così che le varie forme godono di una relativa indipendenza, pur essendo tutte espressione di un comune momento patogenetico. Basti ad es. ricordare che le ecchimosi spontanee si osservano quasi esclusivamente nel sesso femminile e, di solito, in fase sessualmente attiva, mentre nei maschi l'epistassi è propria della fase prepuberale ed immediatamente postpuberale, per scomparire con l'età adulta, salvo a ripresentarsi con l'andropausa. La diversa età di comparsa delle varie forme del complesso mesenchimosico, l'aggravarsi o l'attenuarsi di alcune di esse in determinate fasi della vita, stanno ad indicare accanto al fattore genetico predisponente l'esistenza di altri fattori che possono favorire, ostacolare, localizzare le singole manifestazioni. Fra questi rivestono particolare importanza le variazioni incretorie di quelle ghiandole capaci di indurre a livello dei tessuti di origine mesenchimale un accentuato catabolismo od un deficit di anabolismo proteico. La realizzazione clinica dello stato mesenchimosico è la risultante dell'azione di molteplici fattori, genetici o paratipici, che agendo sulla predisposizione ereditaria ne possono modificare la penetranza e l'espressività. Prestabilite differenze strutturali di sesso (basti pensare alla diversa conformazione nei due sessi del canale inguinale), turbe dell'ontogenesi, modalità e tempo di azione dei fattori endocrini con le loro elettive sedi di azione, traumi fisici ed occupazioni professionali possono in vario modo influenzare l'insorgenza e l'associazione delle varie manifestazioni della diatesi mesenchimosica.

Questa appare quindi come un superamento dello status varicosus di Curtius, sia perchè la tara ereditaria non si liniita alla semplice « displasia della parete venosa » ma interessa diffusamente $\mathrm{i}$ tessuti di origine mesenchimale, sia soprattutto perchè è stato possibile riconoscere e definire molti di quei fattori che ne condizionano la comparsa e ne influenzano l'evoluzione. D'altra parte non sembra che tutte le molteplici forme di « displasia della parete venosa » elencate da Curtius possano far parte della diatesi mesenchimosica, poichè per alcune di esse assumono importanza preminente, accanto all'ipotonia vascolare, altri fattori patogenetici, come ad es. una turbata produzione di sostanze locali di crescenza per alcuni tipi di angiomi, o variazioni distrettuali di circolo (stasi ed iperemia peristatica) per alcune forme di teleangiectasie (Lunedei). A questo proposito sono significativi i risultati di Wagner i quali dimostrano che non esiste una correlazione statisticamente valida fra varici da un lato e teleangiectasie ed angiomi senili dall'altro.

Concludendo quindi, queste nostre ricerche confermano la realtà clinica della diatesi mesenchimosica. L'intercorrelazione che esiste fra alcuni quadri anatomoclinici, quali l'artrosi ad impronta diffusiva, le varici, le ernie, l'epistassi giovanile 
recidivante e le ecchimosi spontanee, deve essere ritenuta come l'espressione di una turba a carattere sistemico e non una combinazione apparente di manifestazioni indipendenti legate fra loro solamente dal progredire dell'età, poichè è stato dimostrato che questa non determina l'associazione dei singoli quadri. Il fattore patogenetico comune condizionante l'insorgenza e l'associazione di queste varie manifestazioni è, con ogni verisimiglianza, da ricercarsi in un'alterazione, condizionata geneticamente, dei tessuti di origine mesenchimale, consistente nella formazione di un connettivo meno atto alla funzione di sostegno e più facilmente soggetto ad usura. I diversi aspetti clinici del complesso mesenchimosico, le differenze nei due sessi e nelle varie epoche della vita sono il risultato del gioco di molteplici fattori che variamente influenzano la ereditaria labilità dei tessuti predetti.

\section{Riassunto}

L'osservazione clinica dimostra che esiste un'associazione fra artrosi ad impronta diffusiva, varici, ernia, epistassi giovanile recidivante ed ecchimosi spontanee. La validità dell'associazione fra questi quadri anatomo-clinici potrebbe essere infirmata se si dimostrasse che l'associazione stessa è dovuta all'influenza dell'età.

Allo scopo di ricercare l'influenza che il fattore età può avere nell'insorgenza e soprattutto nell'associazione di queste manifestazioni è stata compiuta una ricerca su 4 I 3 soggetti, I 56 uomini e 257 donne.

I dati raccolti sono stati elaborati mediante l'impiego del coefficiente di correlazione semplice, del coefficiente di correlazione parziale con eliminazione della variabile età, e dell'analisi fattoriale.

I risultati ottenuti possono essere cosi riassunti:

I. Esiste una correlazione positiva fra i vari quadri anatomo-clinici (tab. 3 ).

2. Ad eccezione dell'artrosi, nessun'altra manifestazione presenta correlazione significativa con l'età (tab. 4).

3. Dopo eliminazione dell'influenza dell'età, la correlazione fra i vari quadri rimane significativa (tab. 5).

4. L'analisi fattoriale mette in evidenza l'esistenza di un fattore comune alle varie manifestazioni, indipendente dall'età (tab. 6).

I risultati di questa ricerca confermano la realtà clinica della diatesi mesenchimosica. Il fattore patogenetico comune condizionante l'insorgenza e l'associazione di queste varie manifestazioni è, con ogni verisimiglianza, da ricercarsi in una turba dei tessuti di origine mesenchimale condizionata in via genetica e responsabile della formazione di un connettivo meno atto alla funzione di sostegno e più facilmente soggetto ad usura. Numerosi fattori, fra i quali primeggiano quelli endocrini, possono variamente influenzare la penetranza e l'espressività del fattore genetico predisponente e quindi l'insorgenza e l'evoluzione della mesenchimosi.

Si sottolinea infine come tale complesso sindromico sia da tenersi assolutamente distinto dall'altro grande gruppo di affezioni del connettivo, le mesenchimopatie reattive o mesenchimiti (impropriamente dette malattie del collageno o collagenosi 
o da taluni addirittura mesenchimosi), il cui carattere schiettamente flogistico chiaramente le differenzia dalla diatesi mesenchimosica (o mesenchimosi eredocostituzionale) della quale il momento patogenetico essenziale risiede in una primitiva alterazione della funzione di sostegno dei connettivi.

\section{Bibliografia}

Beek C. H.: Teleangiectasia papulosa disseminata. Dermatologica, 10 1, 177, 1950.

Boldrini M.: Statistica. Teoria e Metodo. Giuffrè ed., Milano, $195^{8 .}$

Curtius F.: Untersuchungen über das menschliche Venensystem. I Mitt. Dtsch. Arch. Klin. Med., I62, I94, 1928.

- Untersuchungen über das menschliche Venensystem. II Mitt. Dtsch. Arch. Klin. Med., I62, $33^{\circ}, 1928$.

- Untersuchungen über das menschliche Venensystem. III Mitt. Klin. Wschr., 2, $2141,1928$.

- Klinische Konstitutionlehre. J. Springer ver. Berlin, I954.

Fisher A. R. e Yates F.: Statistical Tables. Oliver \& Boyd. London, 1953.

Lenz F.: Die krankhaften Erbanlagen. In Baur-Fischer-Lenz, Menschliche Erblehre und Rassenhygiene, 4 Aufl., Bd. I. München, I936.

Luneder A. e Coll.: Aspetti del problema della localizzazione dei morbi. Relazione al $55^{\circ}$ Congr. Soc. It. Med. interna. Roma, 1954. Pozzi ed. Roma, 1954.

- e Morandi G. A.: I reumatismi distrofici. Relazione all'VIII Congr. Soc. it. Reumatologia. Sirmione, 1954. Reumatismo, 7, suppl. 3, 1955 .

Neri Serneri G. G. c Bartoli V.: Fondamenti genetici della diatesi mesenchimosica. A.Ge.Me.Ge., 7, IoI e 266 , 1958 .

- - e Nicolettr I.: Analisi statistica e considerazioni sull'importanza dell'età per l'associazione di varie manifestazioni dello stato mesenchimosico. Riv. Clin. Med., 58-64, $195^{8 .}$

Nicoletti I. e Magherini G. : L'analisi fattoriale nelle ricerche clinico-terapeutiche. Rass. St. Psichiat., 46, go3, 1957 .

Siemens H. W.: Das Problem der allgemeinen Venenwandschwächen. Med. Klin., I, 797, I937.

Werrz W.: Erkrankunge des Herzens und der Gefässe. In Baur-Fischer-Lenz, Menschliche Erblehre und Rassenhygiene, 5 Aufl., Bd I e 2. Berlin u. München, r940.

WAGNer G.: Zur Methodik des Vergleichs altersabhängiger Dermatosen. (Zugleich korrelationsstatistische Kritik am sog. "Status varicosus 》). Z. menschl. Vererbungs- u. Konstit. lehre, 33, 57, 1955. 


\section{RÉSUMÉ}

L'observation clinique a démontré qu'il existe une association entre arthrose, hernie, varices, épistaxis et ecchymoses spontanées. $\mathrm{La}$ validité de cette association pourrait être infirmée si l'on démontrait que l'association même est due à l'influence ảe l'âge.

Pour évaluer le rôle que l'influence de l'âge peut jouer dans le déclenchement et surtout dans l'ensemble de ces affections, les AA. ont entrepris une recherche sur 413 sujets, 156 hommes et 257 femmes.

Les données recueillies ont été élaborées avec l'application du coefficient de corrélation simple, du coefficient de corrélation partielle avec élimination de l'âge et de l'analyse factorielle.

Les résultats peuvent être ainsi résumés :

1. Une corrélation positive existe entre les différents cadres anatomo-cliniques (tableau $\left.3^{\text {eme }}\right)$.

2. A l'exception de l'arthrose, aucune autre manifestation ne présente une corrélation significative avec l'âge (tableau $4^{\text {eme }}$ ).

3. Après élimination de l'influence de l'âge, la corrélation est encore significative (tableau $\left.5^{\text {emr }}\right)$.

4. L'analyse factorielle démontre l'existence d'un facteur commun à toutes les affections, indépendent de l'àge (tableau $6^{\text {ème }}$ ).

Les résultats de cette recherche confirment la réalité clinique de la diathèse mésenchymosique. Le facteur pathogénétique commun qui réalise le déclenchement et l'association de ces manifestations doit être recherché vraisembla. blement dans un trouble génétique des tissus conjonctifs, responsable de la formation d'un tissu moins propre à la fonction de soutènement et plus sujet à l'usure. Beaucoup de facteurs, entre lesquels les endocriniens occupent la première place, peuvent, d'une façon variée, influencer la pénétrance et l'expressivité génétique et pour cela le déclenchement et l'évolution de la Mésenchymosis.

Les AA. enfin remarquent, une fois encore, que cet ensemble syndromique doit être rigoureusement distingué de l'autre grand ensemble des maladies du conjonctif, c'est-à-dire les mésenchymopathies réactives ou mésenchymitis, dont le caractère franchement phlogistique les distingue de la diathèse mésenchymosique (ou Mésenchymosis hérédo-constitutionnelle), le moment pathogénétique de laquelle est une primitive altération de la fonction de soutènement des tissus conjonctifs. 
Bartoli V., Neri Serneri G. G., Nicoletti I.: Correlazioni ed analisi fattoriale, ecc.

\section{SUMMARY}

The clinical observation demonstrates that Arthrosis, Varicous Veins, Hernia, Epistaxis juvenilis recidivans and spontaneous Ecchymosis appear very frequently associated in a same subject. Nevertheless the validity of this association among these clinical pictures could be invalidated if one could demonstrate the association itself is due to the influence of age.

A research has been accomplished on 413 subjects, 156 men and 257 women, at the purpose of seeking out the influence that age can have on the rising and above all on the association of these manifestations.

The AA. have elaborated the data by use of the common correlation coefficient, of the partial correlation coefficient, with age kept constant, and of the factorial analysis.

The results can be so summarized:

1. there is a positive correlation among the various clinical pictures (table 3).

2. No manifestation shows a significant correlation with age, excepting arthrosis (table 4).

3. After the elimination of the influence of age, the correlation is still significant (table 5).

4. The factorial analysis brings the existence of a factor joint with the various manifesta- tions and indipendent of age into evidence (table 6).

The results of this research strenghten the clinical reality of the mesenchymosic diathesis.

The pathogenetic common factor conditioning the rising and the association of these manifestations is most likely to be sought in changes of the biochemical state of connective tissue wich causes the output of a connective less apt to the support and easier predisposed to wear out. With the genetic factor other situations have therefore a particular interest, first in this group are the endocrines ones, wich effect variously the penetrance and espressivity of the gene and so the unset and evolution of the Mesenchymosis.

The AA. underline, once more, that this syndromic group is to be kept distinctly sep arated from the other large group of diseases of connective tissue i.e. the socalled reactive mesenchymopathic diseases or mesenchymitis (not properly called collagen diseases or collagenosis and by some even mesenchymosis). That is definitively different, because the inflammatory nature, from the mesenchymosic diathesis (or heredo-constitutional Mesenchymosis) wich is due to changes of the supporting function of the tissues of mesenchymal origin. 


\section{ZUSAMMENFASSUNG}

Die klinische Bemerkungen zeigen, dass ein Verhältnis zwischen die verbreitende Arthrosis, die Krampfader, den Bruch, das rückfallige Nasenbluten der Jugend besteht.

Wir könnten dieses Verhältnis falschich bestätigen, wenn man beweisen könnte, dass das Alter dieses Verhältnis bewirkt.

Zur Frage ob das Alter den Grundsatz und überhaupt das Verhältnis dieser Erscheinungen beeinflusst, haben wir 413 Patienten (156 Menschen, 257 Frauen) versucht. Wir haben das Material durch Verwendung der gewönnlichen Korrelationkoeffizienten, der partiellen Korrelationkoeffizienten mit Ausschliessung des veränderlichen Alters und der faktoriellen Analyse ausgearbeitet. Wir können die Ergebnisse als folgende zusammenfassen:

1. Ein bestimmtes Verhältnis besteht zwischen die unterschiedene Erscheinungen ( $\mathrm{Ta}$ belle 3 ).

2. Ausser der Arthrosis, haben die andere Erscheinungen kein ausdruckvolles Verhältnis mit dem Alter (Tabelle 4).

3. Wenn man den Einfluss des Alters ausschliesst, haben die andere Erscheinungen noch ein Verhältnis miteinander (Tabelle 5).

4. Die faktorielle Analyse zeigt, dass die unterschiedene klinische Erscheinungen deut- lich einen gemeinschaftlichen Faktor haben, der von dem Alter nicht abhängig ist (Tabelle 6).

Die Ergebnisse dieser Untersuchung bestätigen die klinische Wirklichkeit der mesenchymosischen Diathese.

Wir müssen wahrscheinlich den gemeinschaftlichen pathogenetischen Faktor, der den Anfang und das Verhältnis dieser Erscheinungen verursacht, in einer genetischen Störung des Bindegewebes, die die Bildung eines wenig schützfähigen und leichter verzehrbaren Bindegewebes beeinflusst, versuchen.

Viele Faktoren, zuerst die Inneredrusen, können veränderlich die Durchdringbarkeit und die Ausdruckfähigkeit des genetischen Faktors und daraus den Anfang und die Entwicklung der Mesenchymose beeinflussen.

Man unterstreicht schliessich nochmals wie das syndromische Komplex (wo die flogistischen Erscheinungen, wenn sie da sind, ein Nebenvorzeichen bilden) auch angesichts der Vererbung von der anderen grossen Gruppe der Krankheiten des Bindegewebes, d.h. die reaktive Mesenchymopathie oder die Mesenchymitis dessen rein-flogistischer Karakter sie von der mesenchymosischen Diathese (oder der vereblichen konstitutionellen Mesenchymose) unterscheidet, welches pathogenetische Hauptmoment in einer primären Änderung der Stützfähigkeit des Gewebes mesenchymaler Ursprung liegt, absolut unterschieden werden soll. 